
\title{
Reseserch Suare \\ Recursive Hilbert transform method: algorithm and convergence analysis
}

\author{
Zhi-Xiang Hu \\ Hefei University of Technology \\ Hui-Yu Zhu \\ Hefei University of Technology \\ Xiao Huang ( $\sim$ huangxiao@ahjzu.edu.cn ) \\ Anhui Jianzhu University \\ Lun-Hai Zhi \\ Hefei University of Technology
}

\section{Research Article}

Keywords: Recursive Hilbert Transform, Bedrosian Theorem, Instantaneous Frequency, Signal Demodulation, Convergence analysis

Posted Date: January 17th, 2022

DOI: https://doi.org/10.21203/rs.3.rs-1238247/v1

License: (1) (1) This work is licensed under a Creative Commons Attribution 4.0 International License. Read Full License 


\section{Recursive Hilbert transform method: algorithm and convergence analysis}

Zhi-Xiang $\mathrm{Hu}^{1}$, Hui-Yu Zhu ${ }^{1}$, Xiao Huang ${ }^{2 *}$, Lun-Hai Zhi ${ }^{1}$

1. Department of Civil Engineering, Hefei University of Technology, Hefei, Anhui Province 23009, China

2. College of Civil Engineering, Anhui Jianzhu University, Hefei, Anhui Province 230601, China

Corresponding author Tel.: 08615609690331

E-mail address: huangxiao@ahjzu.edu.cn (X. Huang)

Keywords: Recursive Hilbert Transform; Bedrosian Theorem; Instantaneous Frequency; Signal Demodulation; Convergence analysis

Abstract: The Hilbert transform (HT) is an important method for signal demodulation and instantaneous frequency (IF) estimation. The modulus of the analytic signal constructed by the HT is regarded as the amplitude, and the derivative of the instantaneous phase of the extracted pure frequency modulation signal is the IF. When the spectrums of the amplitude function and the oscillation term overlap so as not to satisfy the Bedrosian condition, the instantaneous amplitude (IA) and frequency calculated by the Hilbert transform will contain errors. The recursive Hilbert transform (RHT) is an effective method to overcome this problem. The RHT regards the pure frequency modulation signal obtained by the previous HT as a new signal and recursively computes its Hilbert transform until convergence. The final pure frequency modulation signal of the recursive procedure has the same zerocrossing points as the original signal, and its corresponding quadrature error signal vanishes. We emphasize the convergence analysis of the algorithm and study the convergent tendency of the quadrature error signal in each recursive step. The key to the proof is that the discrete Fourier transform value of the quadrature error signal is regarded as a vector, and the length/norm of the vector decreases with the recursion process. Finally, three examples are used to demonstrate the effective application of 
this method in signal demodulation, IF identification and damped vibrating signal analysis, which indicate the application potential of the RHT method in mono-component signal processing.

\section{Introduction}

The IF contains the current state information of the physical system and it is an important monitoring parameter for evaluating whether the state of the system is healthy[1-4]. Therefore, an important issue of non-stationary signal analysis is estimating the IF of the signal. To identify the IF, it is necessary to demodulate the signal in the form of $x(\mathrm{t})=A(t) \cos [\phi(t)]$ and to obtain the derivative of the phase function of the pure frequency modulation signal as the IF. According to the number of components that make up the signal, the general monitored signals fall into two categories: 1) a mono-component signal and 2) a multi-component signal. A single-component signal is also called an intrinsic mode function (IMF), it has good mathematical properties and clear physical meanings. The mathematical characteristics of the amplitude and phase of the IMF are defined in [5]. In general, the amplitude function of a good IMF should be a slow-varying signal, and also the IF should be smooth. Various methods can be used to demodulate the IMFs. For multi-component signals, it is often necessary to decompose the signal first to obtain IMFs. The most famous decomposition method is the empirical mode decomposition (EMD) proposed by Huang et al [6]. The widely used Hilbert-Huang transform (HHT) decomposed the multicomponent signals into a series of IMFs with the EMD method. Then, the HT is utilized to calculate the instantaneous amplitude and IF for each IMF. The EMD has the problem of mode mixing in its use, and the ensemble empirical mode decomposition can partially solve this problem[7,8]. Based on the HT, many signal decomposition methods have been constructed, such as Hilbert vibration decomposition [9,10], iterated Hilbert transform [11,12], analytical mode decomposition $[13,14]$. All of these methods demonstrate the versatility of the HT.

Once the single-component signals are decomposed, the IF of the signals can be calculated. There are many ways to calculate the IF[15], in which the HT is the most popular one. The HT can demodulate 
the signal and extract the amplitude function and phase function [16]. For a real signal, the HT can define its analytical signal and the derivative of the phase function can be regarded as the IF. However, when the amplitude function and pure frequency modulation term do not meet the requirements of the Bedrosian product theorem[17,18], the signal cannot be demodulated correctly using the HT. From the perspective of signal demodulation, HT performs amplitude and phase demodulation at the same time [19]. Thus, in general, the HT cannot give accurate demodulation results. When the HT is used to calculate the IF of a mono-component signal, the difference between the HT of the signal and its quadrature signal is called the quadrature error signal. Nuttall et al. have given the energy calculation formula of the quadrature error signal and pointed out that the energy of the quadrature error signal is not zero for most realistic signals [20]. If the energy of the quadrature error signal is not zero, the IF cannot be accurately calculated using the HT.

This paper introduces a recursive Hilbert transform method, which can extract the instantaneous amplitude/IF when the spectrum of the amplitude function and the spectrum of the oscillating term overlap. The RHT uses the pure frequency modulation signal obtained in the previous step as a new signal, and recursively uses HT to calculate a new pure frequency modulation signal. The RHT method is similar to the normalized Hilbert transform method [21], but it is not necessary to use the extreme value fitting method when calculating the amplitude. The RHT does not require that the amplitude function and the oscillation term strictly satisfy the condition of the Bedrosian product theorem, so it has a larger application range in signal demodulation. The pure frequency modulation signal finally obtained by RHT has the same zero-crossing point as the original signal. The characteristic that the signal zerocrossing point remains unchanged has important physical meanings, which guarantee that the obtained IF can be close to the true IF. Liu et al. have used RHT in conjunction with the synchrosqueezing wavelet transform and analytical mode decomposition to form a combined method for IF identification of low-frequency structures[22]. Ge et al. have used RHT to improve the accuracy of Hilbert square 
demodulation (HSD) and identify the IF of a structure with a nonlinear bolted joint[23]. These recent applications have proven the potential ability for vibrating signal processing.

The early version of the RHT was proposed by Hu and Ren in 2016 [24], but the convergence property of the method has not been studied thoroughly. This paper emphasizes the theoretical analysis on the convergence of RHT. It has been proven that the energy of the quadrature error signal in the recursive process decreases with the increase of the number of recursive steps, so the convergence of RHT can be guaranteed. In the process of proof, the update process of the Fourier transform of the quadrature error signal is regarded as a vector autoregressive process, and the singular values of the coefficient matrix are all less than 1 , so the recursive process can be converged [25,26]. Finally, three examples are used to prove the convergence and application potential of RHT, including exploring the application of RHT in IF estimation, and amplitude/frequency computation of damped free vibrating signal. Due to the robustness and convergence of the RHT, it is expected to be widely used for non-stationary signal processing.

\section{Methods}

\section{$2.1 \mathrm{HT}$}

The Hilbert transform of the signal $x_{0}(\mathrm{t})=A(t) \cos [\phi(t)]$ involves calculation the convolution of $x_{0}(t)$ and $1 / \pi t$, i.e.,

$$
\tilde{x}_{0}(t)=\mathrm{H}\left[x_{0}(t)\right]=\frac{1}{\pi} \mathrm{P} \cdot \int_{-\infty}^{+\infty} \frac{x_{0}(\tau)}{\tau-t} \mathrm{~d} \tau
$$

where $\mathrm{P}$. indicates that the above formula is the principal value integral, both $\tilde{x}$ and $\mathrm{H}[x]$ denote the HT of $x$. The HT is usually used to construct the analytic signal $z_{0}(t)$ as

$$
z_{0}(t)=x_{0}(t)+j \cdot \tilde{x}_{0}(t)=A_{0}(t) \exp \left[-j \phi_{0}(t)\right]
$$


In this definition, the imaginary part of the analytic signal is the Hilbert transform of the real part, and the modulus and the phase of the analytic signal can be computed by

$$
\begin{aligned}
& A_{0}(t)=\sqrt{x_{0}^{2}(t)+\tilde{x}_{0}^{2}(t)} \\
& \phi_{0}(t)=\operatorname{atan}\left[\tilde{x}_{0}(t) / x_{0}(t)\right]
\end{aligned}
$$

Therefore, the original signal can be expressed by the product of the modulus of the analytic signal and a pure frequency modulation signal

$$
x_{0}(t)=\mathrm{A}_{0}(t) \cos \left[\phi_{0}(t)\right]
$$

Using the phase $\phi_{0}(t)$, one can derive the IF as

$$
\omega_{0}(t)=\dot{\phi}_{0}(t)
$$

The above steps are the process of using HT to demodulate the mono-component signal and calculate the IF. When the frequency spectrum of the amplitude function and the oscillation term meet the Berdosian condition, the original signal can be demodulated accurately by the HT [17]. However, in general, the spectrum of the amplitude function may overlap with the oscillation term. Thus $A_{0}(t)$ may not equal to $A(t)$, and the demodulated amplitude and phase by the HT will contain an obvious error. To achieve successful signal demodulation with the HT, there are rigorous conditions for monocomponent signals. Therefore, it is necessary to study demodulation algorithms for a wider range of signals.

\subsection{RHT}

Regard the pure frequency modulated signal obtained by the HT, $x_{1}(t)=\cos \left[\phi_{0}(t)\right]$, as a new signal, and perform Hilbert transform on $x_{1}(t)$ to get a new amplitude function and pure frequency modulation signal. The recursive formula is 


$$
x_{k+1}(t)=\cos \left[\phi_{k}(t)\right]=x_{k}(t) / \sqrt{x_{k}^{2}(t)+\tilde{x}_{k}^{2}(t)}
$$

where $\tilde{x}_{k}(t)$ is the Hilbert transform of $x_{k}(t)$, and the recursive step $k=0,1,2, \cdots, K$. When $k=0$, the calculation is the original Hilbert transform, and $K$ is the total step number. In each step, the corresponding amplitude function $A_{k}$ and phase function $\phi_{k}$ can be obtained by

$$
\begin{aligned}
A_{k}(t) & =\sqrt{x_{k}^{2}(t)+\tilde{x}_{k}^{2}(t)} \\
\phi_{k}(t) & =\operatorname{atan}\left[\tilde{x}_{k}(t) / x(t)\right]
\end{aligned}
$$

With the increase of the recursive step, the shape of the new pure frequency modulated signal $\cos \left[\phi_{k}(t)\right]$ changes gradually. The quadrature error signal [20] of step $k$ is defined by

$$
e_{k}(t)=\mathrm{H}\left\{\cos \left[\phi_{k}(t)\right]\right\}-\sin \left[\phi_{k}(t)\right]
$$

The quadrature error signal will decrease as the number of recursions increases. The energy of the quadrature error signal $\left\|e_{k}(t)\right\|$ can be used as the index for the convergence judgment. Also, the variation of the pure frequency modulation signal obtained in the recursive process can be used as the index for the convergence judgment. The variation is given by $v_{k}(t)=x_{k+1}(t)-x_{k}(t)$. If $\left\|v_{k}(t)\right\|$ trends to 0 , the pure frequency modulation signal obtained by the recursive process no longer changes, and the calculation can be stopped. The calculation process of the RHT is shown in Figure 1, wherein the infinitesimal number $\epsilon$ is set to be $10^{-15}$ in this work. The convergence of the recursive process will be analyzed in the next section. Denote $x_{K+1}(t)=\cos \left[\phi_{K}(t)\right]$ as the final pure frequency modulation signal whose Hilbert transform equals to its quadrature signal, i.e.,

$$
\mathrm{H}\left[x_{K+1}(t)\right]=\mathrm{H}\left\{\cos \left[\phi_{K}(t)\right]\right\}=\sin \left[\phi_{K}(t)\right]
$$

And the IF of the signal $\cos \left[\phi_{K}(t)\right]$ is

$$
\omega_{K}(t)=\dot{\phi}_{K}(t)
$$


Considering all the recursive steps, the original signal can be expressed as the product of the amplitude functions and the final pure frequency modulation signal.

$$
x_{0}(t)=\left(\prod_{k=1}^{K} A_{k}\right) \cos \left[\phi_{K}(t)\right]
$$

It can be seen that the RHT can decompose the original signal into two parts, the amplitude function and the pure frequency modulation signal. Obviously, the final pure frequency modulation signal has the following properties:

(1) $\cos \left[\phi_{K}(t)\right]$ has the same zero-crossing points with the original signal $x_{0}(t)$.

(2) $\phi_{K}(t)$ eauals to $\phi(t)$ at zero-crossing points. According to the Lagrange mean value theorem, there is at least one point between the two zero-crossing points at which the instantaneous frequency is true.

(3) The complex signal $z_{K}(t)=\cos \left[\phi_{K}(t)\right]+j \cdot \sin \left[\phi_{K}(t)\right]$ is an analytic signal.

The true IF of the original signal $x_{0}(t)$ is $\omega(t)=\dot{\phi}(t)$, and the computed IF given by the RHT is $\omega_{K}(t)=\dot{\phi}_{K}(t)$. According to property (2), for a vibration signal with a slow-varying IF, the RHT can be used to estimate an accurate IF. The natural frequencies of many actual systems change slowly over time, so the RHT has a broad application prospect. 


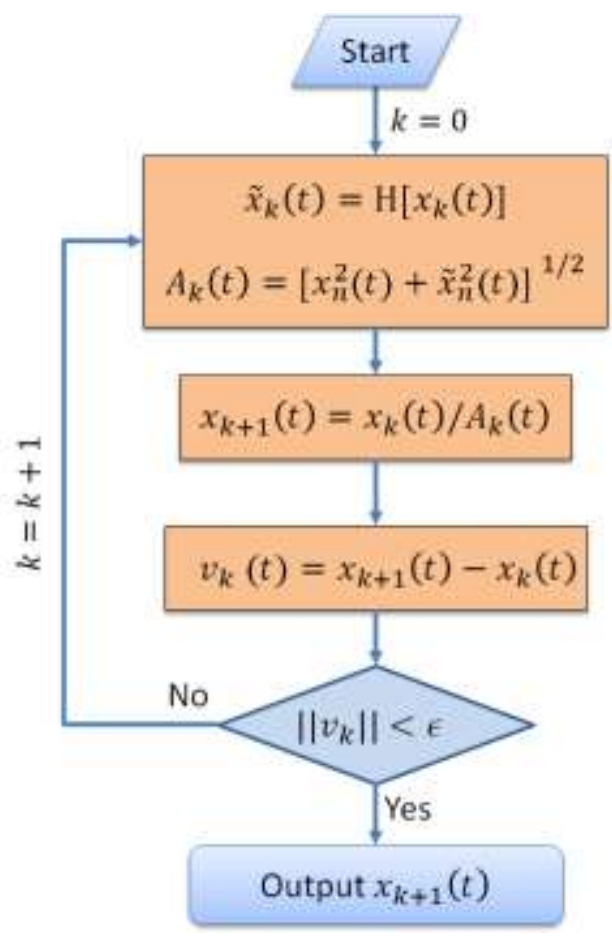

Fig1. Algorithm flow chart of the RHT

\subsection{Convergence analysis}

The quadrature signal of $\cos \phi_{k}$ is $\sin \phi_{k}$, and the corresponding quadrature error signal $e_{k}$ is computed by Eq.(10). Here the phase $\phi_{k}(t)$ is a function of time $t$, it is abbreviated as $\phi_{k}$ for concise, other functions are treated in the same way. When $e_{k} \neq 0$, the complex signal $\cos \phi_{k}+i \sin \phi_{k}$ (corresponding to the pure frequency modulated signal $\cos \phi_{k}$ ) is not an analytic signal. Meanwhile, the complex signal $\cos \phi_{k}+i \mathrm{H}\left[\cos \phi_{k}\right]$ constructed by the HT is an analytic signal, and its phase function is

$$
\phi_{k+1}=\operatorname{atan} \frac{\mathrm{H}\left[\cos \phi_{k}\right]}{\cos \phi_{k}}
$$

Thus we can obtain a new pure frequency modulation signal $\cos \phi_{k+1}$, its corresponding quadrature error signal is

$$
e_{k+1}(t)=\mathrm{H}\left[\cos \phi_{k+1}\right]-\sin \phi_{k+1}
$$


To study the evolution of the quadrature error signal as the recursive step increases, the reciprocal of the amplitude $A_{k}$ is first expanded into Taylor series, i.e.,

$$
\frac{1}{A_{k}}=\frac{1}{\sqrt{\cos ^{2} \phi_{k}+\left(\mathrm{H} \cos \phi_{k}\right)^{2}}}=1-\sin \phi_{k} \cdot e_{k}+O\left(e_{k}^{2}\right)
$$

where $O\left(e_{k}^{2}\right)$ represents the second-order infinitesimal of the quadrature error signal, and the analysis is restricted to terms of the first order of $e_{k}$. With this expansion, one can obtain

$$
\begin{gathered}
\cos \phi_{k+1}=\frac{\cos \phi_{k}}{A_{k}}=\cos \phi_{k}-\frac{1}{2} \sin 2 \phi_{k} \cdot e_{k}+O\left(e_{k}^{2}\right) \\
\sin \phi_{k+1}=\frac{\mathrm{H}\left[\cos \phi_{k}\right]}{A_{k}}=\sin \phi_{k}+\frac{1}{2} e_{k}+\frac{1}{2} \cos 2 \phi_{k} \cdot e_{k}+O\left(e_{k}^{2}\right)
\end{gathered}
$$

Hence, substitute Eq.(17) and (18) into Eq.(15), the relationship of $e_{k+1}$ and $e_{k}$ can be established as

$$
e_{k+1}=\frac{1}{2} e_{k}-\frac{1}{2}\left(\mathrm{H}\left[\sin 2 \phi_{k} \cdot e_{k}\right]+\cos 2 \phi_{k} \cdot e_{k}\right)+O\left(e_{k}^{2}\right)
$$

From this equation, the effect of the recursive progress to the quadrature error signal can be investigated. Let

$$
e_{k}=e_{L, k}+e_{H, k}=\sum_{1}^{l} c_{j} \cos \phi_{e, j}+\sum_{l+1}^{M} c_{j} \cos \phi_{e, j}=\sum_{1}^{M} c_{j} \cos \phi_{e, j}
$$

where $e_{L, k}=\sum_{1}^{l} c_{j} \cos \phi_{e, j}$ and $e_{H, k}=\sum_{l+1}^{M} c_{j} \cos \phi_{e, j}$ are low-frequency components and highfrequency components contained in $e_{k}$, respectively. And $M$ is the total number of the components. They satisfy the Bedrosian product theorem below

$$
\begin{gathered}
\mathrm{H}\left[\sin 2 \phi_{k} \cdot e_{L, k}\right]=\mathrm{H}\left[\sin 2 \phi_{k}\right] \cdot e_{L, k} \approx-\cos 2 \phi_{k} \cdot e_{L, k} \\
\mathrm{H}\left[\sin 2 \phi_{k} \cdot e_{H, k}\right]=\sin 2 \phi_{k} \cdot \mathrm{H}\left[e_{H, k}\right]
\end{gathered}
$$


Substituting Eq.(21) into Eq.(20) one finds that the low-frequency component in $e_{k}$ is eliminated. Therefore, the update formula of the quadrature error signal can be expressed as

$$
e_{k+1} \approx \frac{1}{2} e_{k}-\frac{1}{2} \sum_{l+1}^{M} c_{l} \cos \left(\phi_{e, l}-2 \phi_{k}\right)
$$

With Eq.(23), the convergence trend of the quadrature error signal can be guaranteed. The frequency of the high-frequency components in the quadrature error signal continues to decrease $\left(2 \dot{\phi}_{k}\right.$ in each recursive step, and multiplied by -1/2). These components become low-frequency components after their frequencies are less than $2 \dot{\phi}_{k}$. When the quadrature error signal does not contain high-frequency components, the signal amplitude will continue to halve as the recursive step increases, and the energy of $e_{k}$ will continue to attenuate.

To quantitatively analyze the changes of the quadrature error signal, the analysis is performed for a signal with unchanged IF ( $\dot{\phi}_{k}=\omega$ is a constant number). Under this condition, the Fourier transform of the quadrature error signal can be derived as

$$
F_{k+1}\left(\omega_{j}\right) \approx \frac{1}{2} F_{k}\left(\omega_{j}\right)-\frac{1}{2} F_{k}\left(\omega_{j}+2 \omega\right)
$$

The evolution of the Fourier coefficient of $e_{k}$ is illustrated in Fig.2, where the dimension of the circles depicts the absolute value of the Fourier coefficients at each frequency point, and the colour of the circles represents its sign (Blue denote positive, orange denote negative). After each recursion, the amplitude of high-frequency components is reduced to $1 / 2$, and new signal components are produced at the frequency point $\dot{\phi}-2 \omega$ (and multiplied by -1/2). The low-frequency components are simply reduced by half. Since there is a region wherein only signal energy attenuation occurs, as shown in Fig.2, one can qualitatively determine the energy attenuation trend of the quadrature error signal as the recursive step increases. 


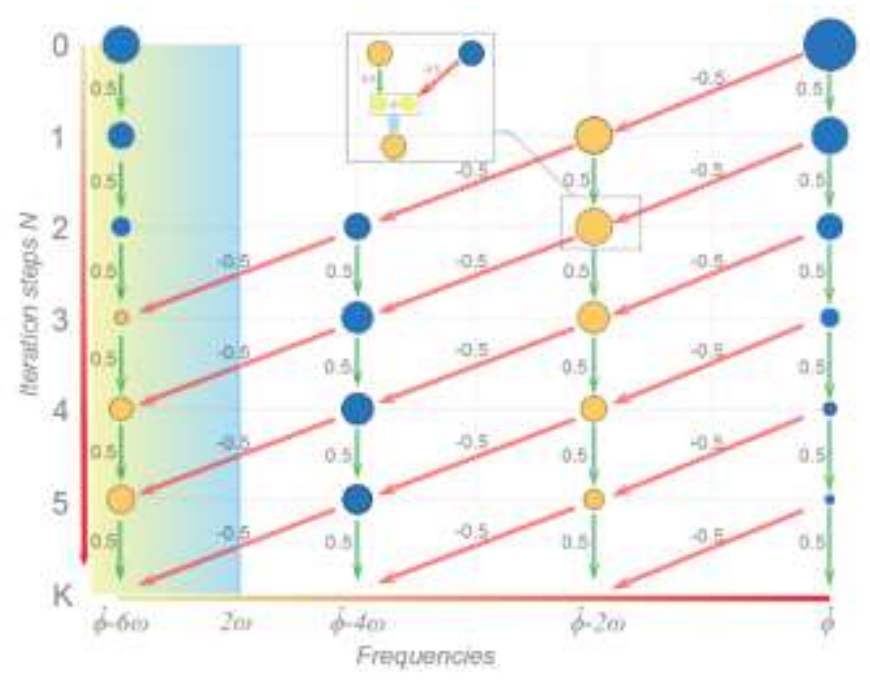

Fig.2 Evaluation diagram of the Fourier coefficient of the quadrature error signal

\begin{tabular}{|c|c|c|c|c|c|c|c|c|c|c|}
\hline & 1 & 2 & 3 & 4 & 5 & 6 & 7 & 8 & 9 & 10 \\
\hline 1 & 0.50 & & & & -0.50 & & & & & \\
\hline 2 & & 0.50 & & & & -0.50 & & & & \\
\hline 3 & & & 0.50 & & & & -0.50 & & & \\
\hline 4 & & & & 0.50 & & & & $-0,50$ & & \\
\hline 5 & & & & & 0.50 & & & & -0.50 & \\
\hline 6 & & & & & & 0.50 & & & & -0.50 \\
\hline 7 & & & & & & & 0.50 & & & \\
\hline 8 & & & & & & & & 0.50 & & \\
\hline 9 & & & & & & & & & 0,50 & \\
\hline 10 & & & & & & & & & & 0.50 \\
\hline
\end{tabular}

Fig.3. An example of matrix A with $n_{2 \omega}=4$ and $M=10$

The changing of the Fourier transform value of the quadrature error signal can be regarded as a vector autoregressive process[25],

$$
\boldsymbol{F}_{k+1} \approx \mathbf{A} \boldsymbol{F}_{k}
$$

where $\boldsymbol{F}_{k}$ is the vector formed by the Fourier transform coefficients at discrete frequency points, and the matrix $\mathbf{A}$ is the coefficient matrix. The elements in $\mathbf{A}$ are given by

$$
\mathbf{A}_{i j}=\left\{\begin{array}{rc}
0.5 & \text { if } j=i \\
-0.5 & \text { if } j=i+n_{2 \omega} \\
0 & \text { else }
\end{array}\right.
$$


where $n_{2 \omega}$ is the number of the frequency point $2 \omega$. In Fig.3, a simple example of the matrix $\mathbf{A}$ is shown with $n_{2 \omega}=4$ and total frequency point number $M=10$. In general, the matrix $\mathbf{A}$ has the form below:

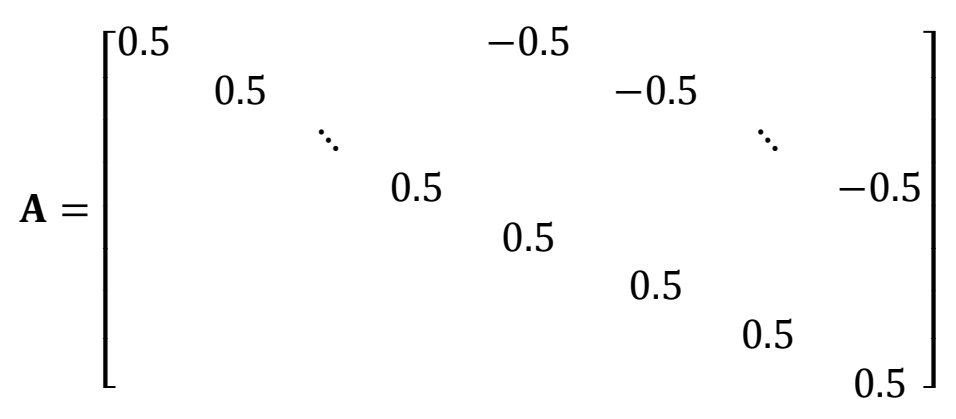

Performing singular value decomposition to $\mathbf{A}$, we have $\mathbf{A}=\mathbf{U S V}^{T}$, where both $\mathbf{U}$ and $\mathbf{V}$ are unitary matrices, $\mathbf{S}$ is a diagonal matrix that contains the singular values of $\mathbf{A}$. It is proved that all singular values of matrix $\mathbf{A}$ are less than 1 , thus $\mathbf{S}_{j j}<1, j=1,2, \ldots, M$ (See Appendix A for the proof). Now for a vector $\boldsymbol{X} \in \boldsymbol{C}^{M \times 1}$,

$$
\|\mathbf{S} \boldsymbol{X}\|=\sqrt{\sum_{j=1}^{M} \boldsymbol{S}_{j j}^{2}\left|\boldsymbol{X}_{j}\right|^{2}}<\sqrt{\sum_{j=1}^{M}\left|\boldsymbol{X}_{j}\right|^{2}}=\|\boldsymbol{X}\|
$$

where $\|\mathbf{\square}\|$ means the Euclidean norm of $\mathbf{\square}$. This shows that the norm (length) of a vector decreases after multiplied by $\mathbf{S}$. Meanwhile, any vector multiplied by a unitary matrix remains its norm (length) unchanged, i.e., $\left\|\mathbf{V}^{T} \boldsymbol{X}\right\|=\|\boldsymbol{X}\|,\|\boldsymbol{U} \boldsymbol{X}\|=\|\boldsymbol{X}\|$. With these properties, we have

$$
\begin{gathered}
\left\|\mathbf{A} \boldsymbol{F}_{k}\right\|=\left\|\mathbf{U S V}^{T} \boldsymbol{F}_{k}\right\|=\left\|\mathbf{S} \mathbf{V}^{T} \boldsymbol{F}_{k}\right\| \\
\left\|\mathbf{S} \mathbf{V}^{T} \boldsymbol{F}_{k}\right\|<\left\|\mathbf{V}^{T} \boldsymbol{F}_{k}\right\|=\left\|\boldsymbol{F}_{k}\right\|
\end{gathered}
$$

Finally, combining the above two formulas we have

$$
\left\|\mathbf{A} \boldsymbol{F}_{k}\right\|<\left\|\boldsymbol{F}_{k}\right\|
$$


It can be seen that after one recursion, the norm of the vector corresponding to the Fourier transform of the quadrature error signal becomes smaller, which indicates that the energy contained in the quadrature error signal decreases with the recursive progress. This certifies the convergence of the RHT.

\section{Experiments and result discussions}

In this section, three examples are represented to demonstrate the performance of the RHT algorithm. In the first example, it is shown that the RHT can give good demodulation results for signals that violate the Bedrosian condition. In the second example, the RHT is used to estimate the IFs of time-varying signals, and its performance is compared to the HT and the Teager operator method [15]. In the third example, the RHT is used for processing the damped vibrating signal processing. These examples are helpful to understand the characteristics of RHT. The convergence of the RHT is also validated in the examples.

\subsection{RHT for signals that violate the Bedrosian condition}

In this example, we focus on a simple AM signal $x(t)=A(t) \cos [\phi(t)]$, wherein $\phi(t)=\omega t+\varphi_{0}$, the frequency $\omega$ is constant, and $\varphi_{0}$ is the initial phase. The amplitude function satisfies $A(t)>0$. To correctly demodulate this signal with HT, the highest frequency of the components in the amplitude should not be greater than $\omega$. This limitation is often not met in actual signal processing, making the HT demodulation results contain errors. A well-designed amplitude function that contains high-frequency components is considered, as shown in Fig.4. The carrier frequency of the oscillation term is $\omega=20 \pi$ ( $f=10 \mathrm{~Hz}$ ), while the highest frequency of the components of $A(t)$ is $\omega_{h}=40 \pi\left(f_{h}=20 \mathrm{~Hz}\right)$. Therefore, the $A(t)$ fluctuates more intensely than the oscillation term itself. The Fourier amplitude spectrum of the oscillation term $\cos \left(\omega t+\varphi_{0}\right)$ and its amplitude $A(t)$ are both shown in Fig.4b. We can inspect that $A(t)$ contains high-frequency harmonic components whose frequency are greater than 10 Hz. The amplitude is generated by 


$$
A(t)=1+\sum_{l=1}^{\omega_{h} / \Delta \omega} a_{l} \cos \left(l \cdot \Delta \omega \cdot t+\varphi_{l}\right)
$$

where $\Delta \omega=\frac{2 \pi}{T}$ is the frequency resolution of the discrete Fourier transform and $T$ is the total signal time; $a_{l}$ and $\varphi_{l}$ are amplitude and initial phase of each harmonic component, respectively. Their values are generated from uniform distributions, $a_{l} \sim U\left(0, \gamma \frac{\Delta f}{f_{h}}\right), \varphi_{l} \sim U(0,2 \pi)$. Thus the extreme values of $A(t)$ are also random variables. The degree of fluctuation is controlled by the parameter $\gamma$. In the first simulation, we choose $\gamma=3$.

To compare the error of the obtained pure frequency modulation signals more clearly, the formula for calculating the demodulation error is given as follows

$$
\begin{gathered}
r_{\mathrm{HT}}(t)=\cos \left[\phi_{0}(t)\right]-\cos [\phi(t)] \\
r_{\mathrm{RHT}}(t)=\cos \left[\phi_{K}(t)\right]-\cos [\phi(t)]
\end{gathered}
$$

where $r_{\mathrm{HT}}(t)$ and $r_{\mathrm{RHT}}(t)$ denote the error of the HT and the error of the RHT, respectively.

Both the HT and the RHT are used to demodulate the signal $x(t)$. The pure frequency modulation signal obtained by the $\mathrm{HT}$ is $\cos \left[\phi_{0}(t)\right]$. It is compared to the true oscillation term $\cos (\omega t)$ in Fig. 5a. A distinct difference between $\cos \left[\phi_{0}(t)\right]$ and $\cos (\omega t)$ can be found. The amplitude computed by the HT is plotted in Fig. 5b, which is different from the original amplitude. The pure frequency modulation signal and amplitude obtained by the RHT method are shown in Fig. 6. It is found that the resulting pure frequency modulation signal $\cos \left[\phi_{R}(t)\right]$ is almost identical with $\cos (\omega t)$; the demodulated amplitude is also almost identical with true amplitude. The error $r_{\mathrm{HT}}(t)$ and $r_{\mathrm{RHT}}(t)$ are shown in Fig. 7a and Fig. 7b, respectively. The former is remarkable, while the latter is a negligible numerical calculation error. The comparison of the results of the HT and the RHT shows that the latter is more robust for demodulating signals. 

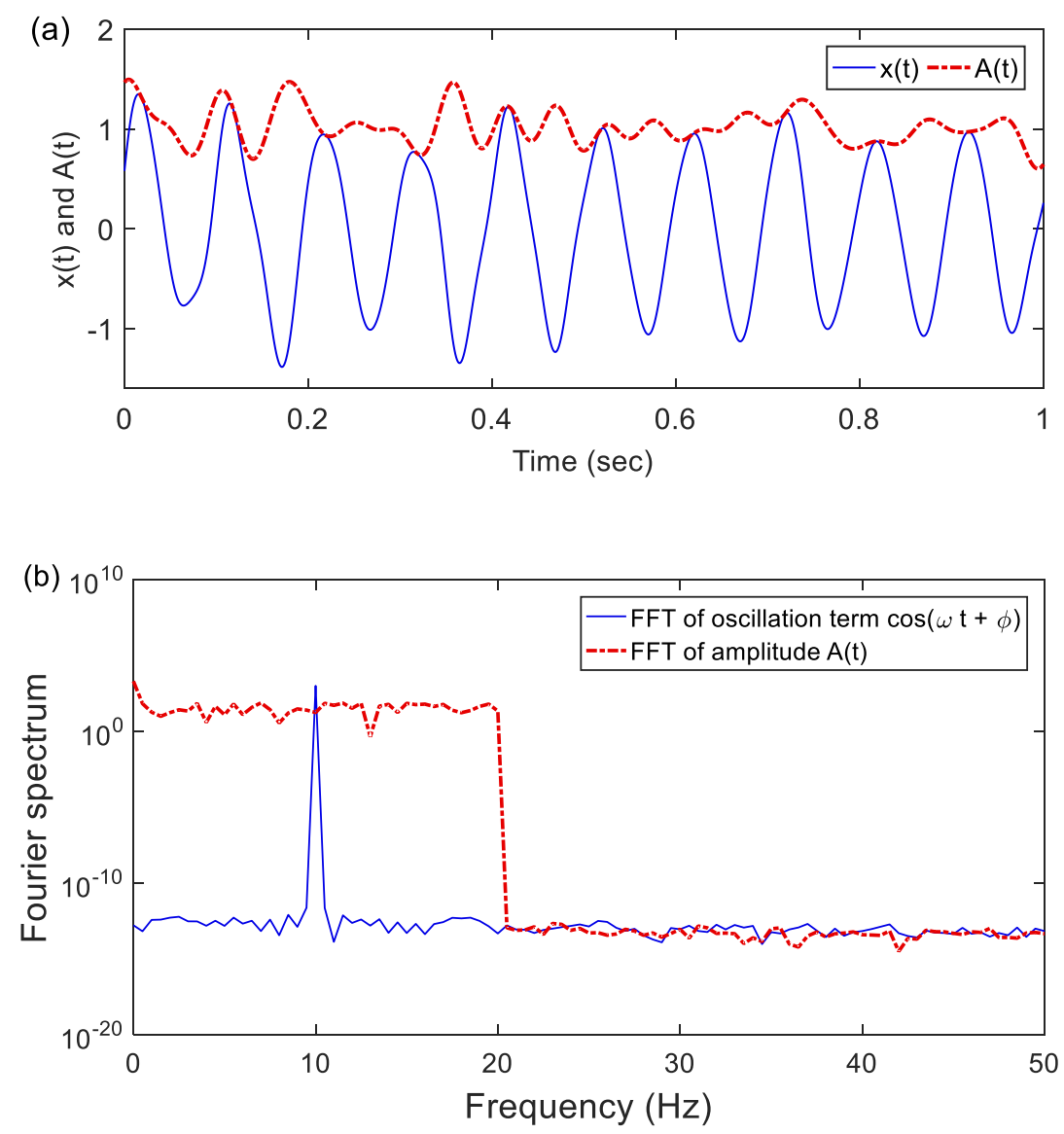

Fig. 4. The AM signal $x(t)$ and its amplitude $A(t)$. (a): waveform of and amplitude; (b): The Fourier amplitude of the amplitude oscillation term and the amplitude

The evolution of the quadrature error signal $e_{k}(t)$ and the variation $v_{k}(t)$ of the pure frequency modulation signal are shown in Fig. 8. With the increase of the recursive step, the amplitude of the quadrature error signal gradually decreases. As shown in Fig. 8a, the $e_{k}(t)$ approximately decreases by $1 / 2$ after each recursion, which indicates that the quadrature error signal mainly contains low-frequency components. The trend of the norm of the quadrature error signal is shown in Fig. 8b, its energy is decreased to below $1 \mathrm{e}-10$ after 30 steps. The variations $v_{k}(t)$ of different recursive step are shown in Fig. 8c. The trend of the energy of the variation, $\left\|v_{k}(t)\right\|$, is shown in Fig. 8d. After about 50 recursions, both $\left\|v_{k}(t)\right\|$ and $\left\|e_{k}(t)\right\|$ approximate to zero. This case is a typical demonstration of RHT's convergence characteristics. 

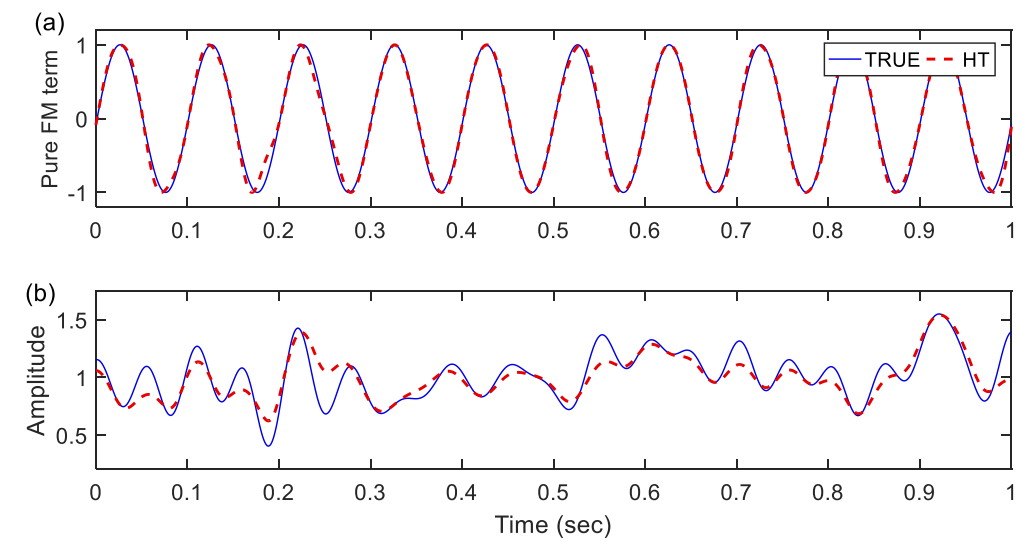

Fig. 5. Results obtained by the HT. (a): Pure frequency demodulation signal result; (b): Amplitude function result.
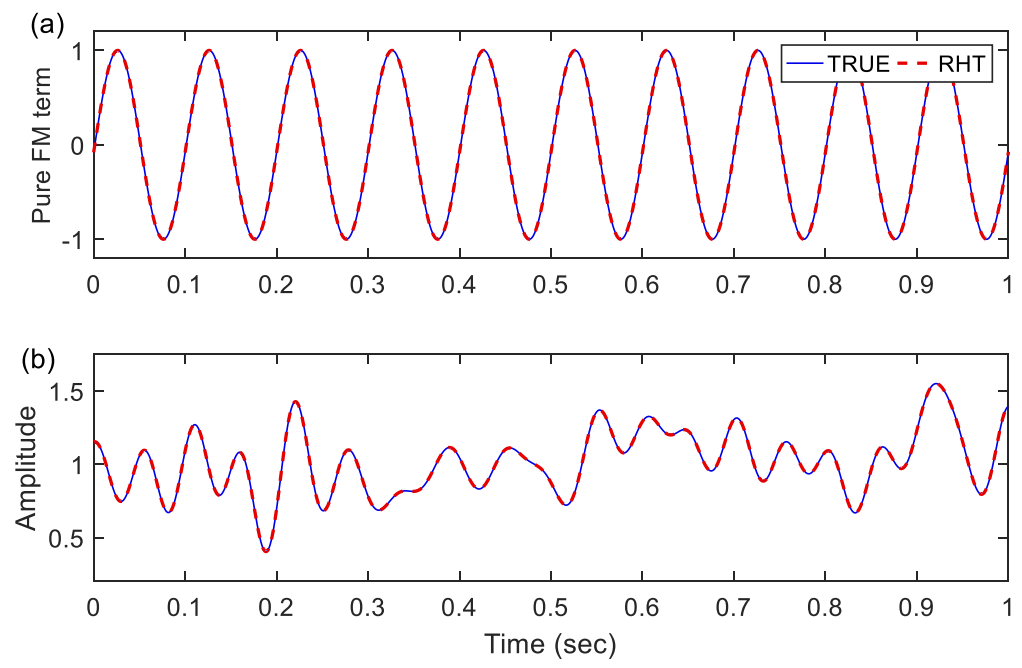

Fig. 6. Results obtained by the RHT. (a): Pure frequency demodulation signal result; (b): Amplitude function result. 

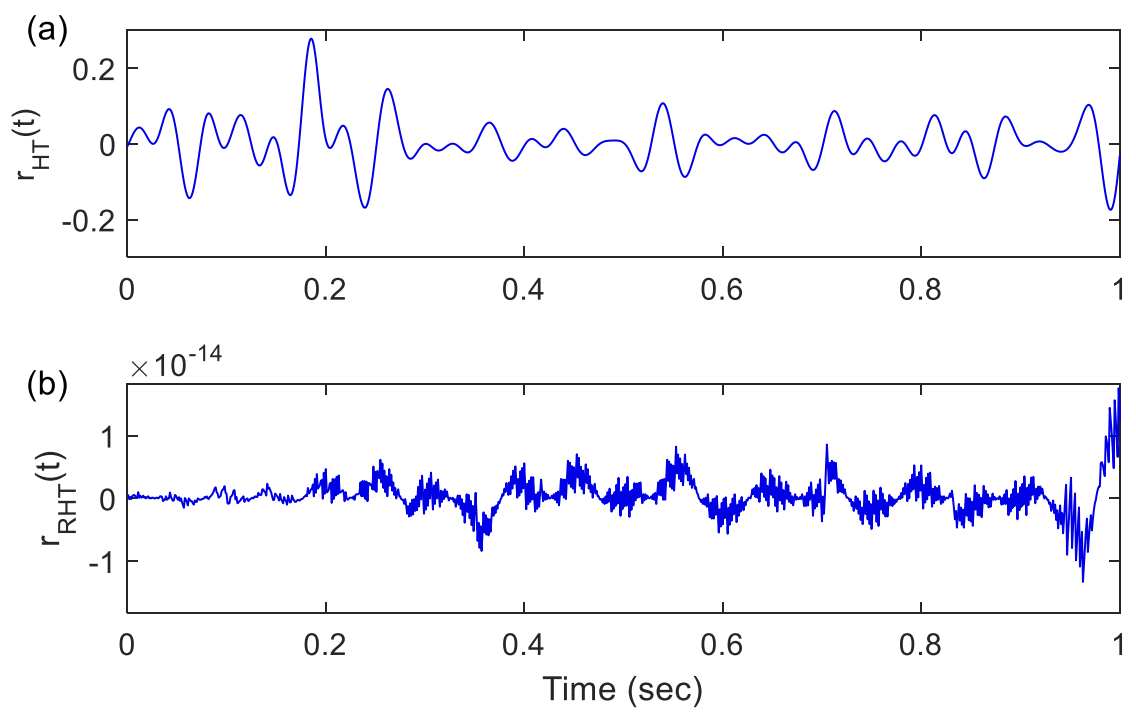

Fig. 7. Demodulation errors for oscillation term. (a): $r_{\mathrm{HT}}(t) ;(b): r_{\mathrm{RHT}}(t)$.
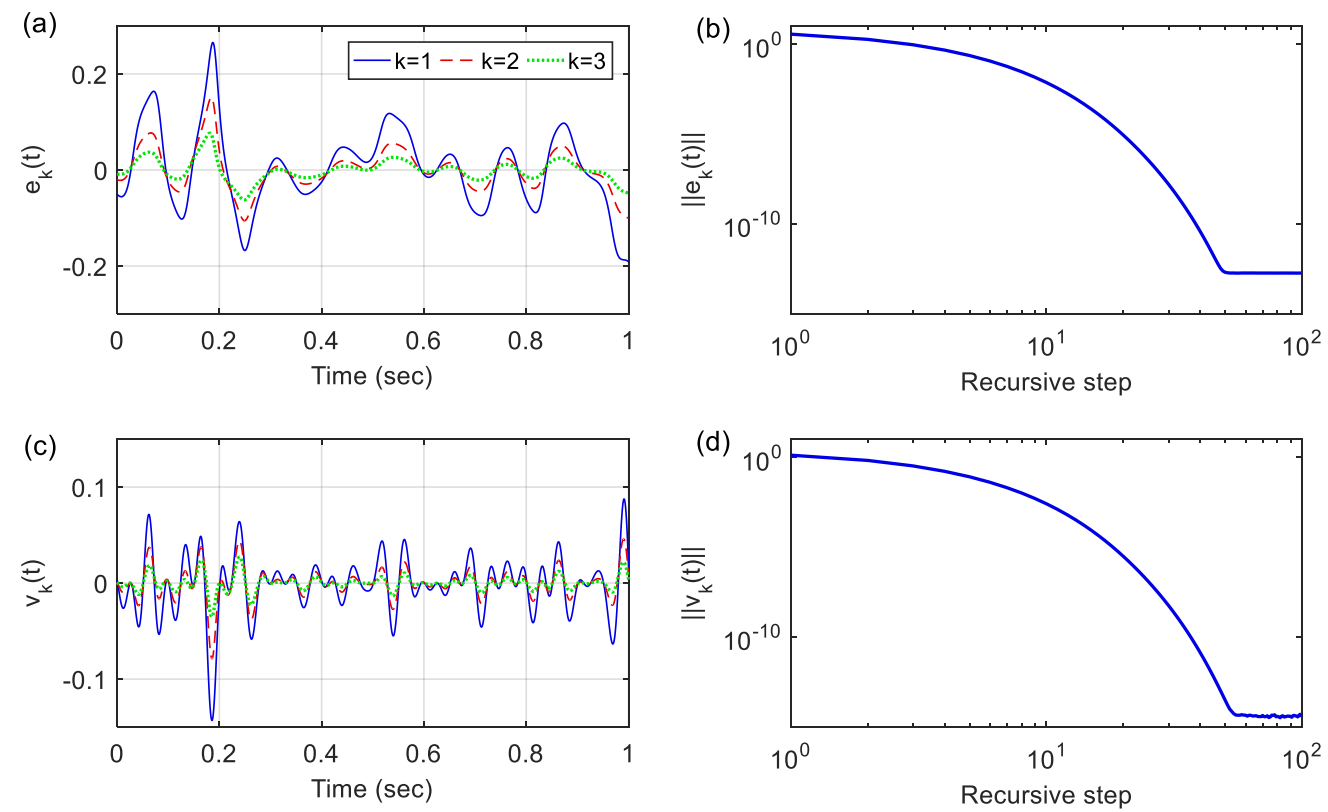

Fig. 8 Convergence trend of the RHT for demodulation of $x(t)$. (a): $e_{k}(t) ;(\mathrm{b}):\left\|e_{k}(t)\right\| ;(\mathrm{c}): v_{k}(t) ;(\mathrm{d})$ : $\left\|v_{k}(t)\right\|$.

To answer the question of whether the convergent results are unique, the Monte-Carlo (MC) method is implemented to analyze $N_{\mathrm{m}}=1000$ sets generated signals. In each MC simulation, the amplitude functions are generated by Eq.(31) with $\gamma=2$. Let $x_{R, m}(t)=\cos \left[\phi_{R, m}(t)\right]$ denote the RHT result of the $m^{\text {th }}$ simulation, $\overline{x_{R}}(t)$ denote the mean value of all results. With different amplitude functions, we 
expect that the obtained pure frequency modulation signals are identical. To represent the variance of the pure frequency modulation signal obtained in the Monte-Carlo simulations, an index is defined as

$$
D=\sqrt{\frac{1}{N} \sum_{j=1}^{N} \sigma^{2}\left(t_{j}\right)}
$$

where $\sigma^{2}\left(t_{j}\right)$ is the result's variance at time $t_{j}$, given by

$$
\sigma^{2}\left(t_{j}\right)=\frac{1}{N_{\mathrm{m}}-1} \sum_{m}^{N_{\mathrm{m}}}\left[x_{R, m}\left(t_{j}\right)-\overline{x_{R}}\left(t_{j}\right)\right]^{2}
$$

The index $D$ will decrease to 0 as the recursive step increases if the result of $\cos \left[\phi_{R}(t)\right]$ is unique. The decreasing trend of the index $D$ is shown in Fig. 9. The value of index $D$ keeps decreasing in the first 40 steps of recursion, which demonstrates the convergence property of the RHT method. In the last several steps, the value of $D$ is generally stable yet its magnitude is very small (below $10^{-14}$ ). Ignoring the numerical calculation error, it can be concluded that for mono-component signals, the result of the pure frequency modulation signal demodulated by the RHT is unique. This trend ensures the stability of RHT for signal demodulation.

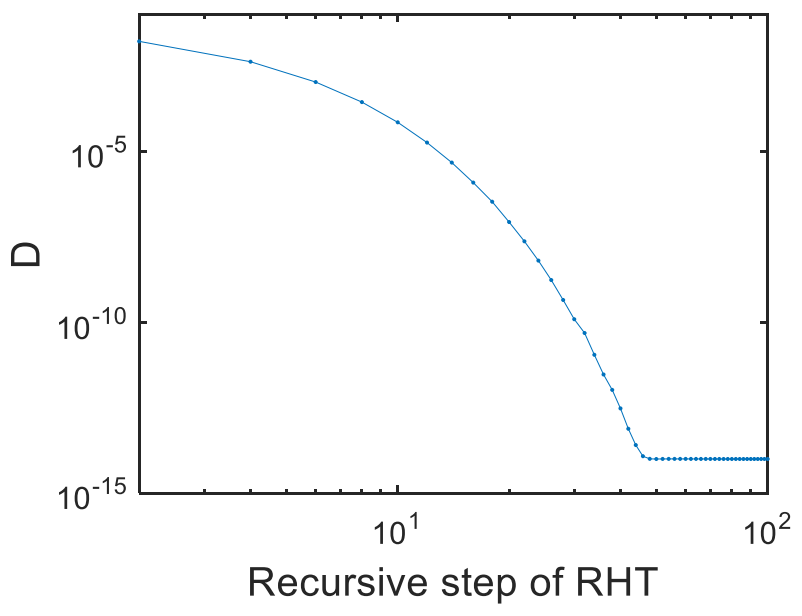

Fig. 9. The trend of index $D$

It should be noted that the above results are obtained when the sampling frequency is $1000 \mathrm{~Hz}$. Since the RHT has the characteristic of keeping the zero-crossing position unchanged, the increase of the 
sampling frequency benefits the improvement of calculation accuracy. In Fig. 10, the values of $D$ for different sampling frequencies are shown. The horizontal axis in the figure is the sampling frequency of the simulated signal, and the vertical axis is the index $D$. In the calculation, the number of recursions $R=100$ and amplitude control parameter $\gamma=2$. And for each sampling frequency, $1000 \mathrm{MC}$ simulations are performed. Then the corresponding uniqueness index $D$ is counted. When the sampling frequency is low, there are remarkable differences in the obtained demodulated pure frequency modulation signals when different amplitude functions are generated in the MC simulation. As the sampling frequency increases, the index $D$ quickly decreases. When the sampling frequency reaches $600 \mathrm{~Hz}$, the index $D$ reaches a stable small value less than $10^{-14}$. This shows that increasing the sampling frequency benefits the performance of RHT.

To understand the effect of sampling frequency on the uniqueness of the result, we should inspect the characteristic of the discrete signal. Suppose $x_{r}\left(t_{L}\right)$ and $x_{r}\left(t_{L}+\Delta t\right)$ are values corresponding to the left and right sides of a zero-crossing point. The zero-crossing time can be approximately estimated by

$$
t_{z p}=t_{L}+\Delta t \cdot \frac{\left|x_{r}\left(t_{L}\right)\right|}{\left|x_{r}\left(t_{L}\right)\right|+\left|x_{r}\left(t_{L}+\Delta t\right)\right|}
$$

Since the zero-crossing position changes within the range of $\Delta t$, at a lower sampling frequency, the obtained waveforms are more unconstrained. Thus the results are not unique. The higher the sampling frequency, the smaller the $\Delta t$, thus the RHT can accurately capture the true position of the zero-crossing points, and the demodulated waveforms are closer to the real waveform. Therefore, a high sampling frequency should be required for the RHT to accurately demodulate the signal. 


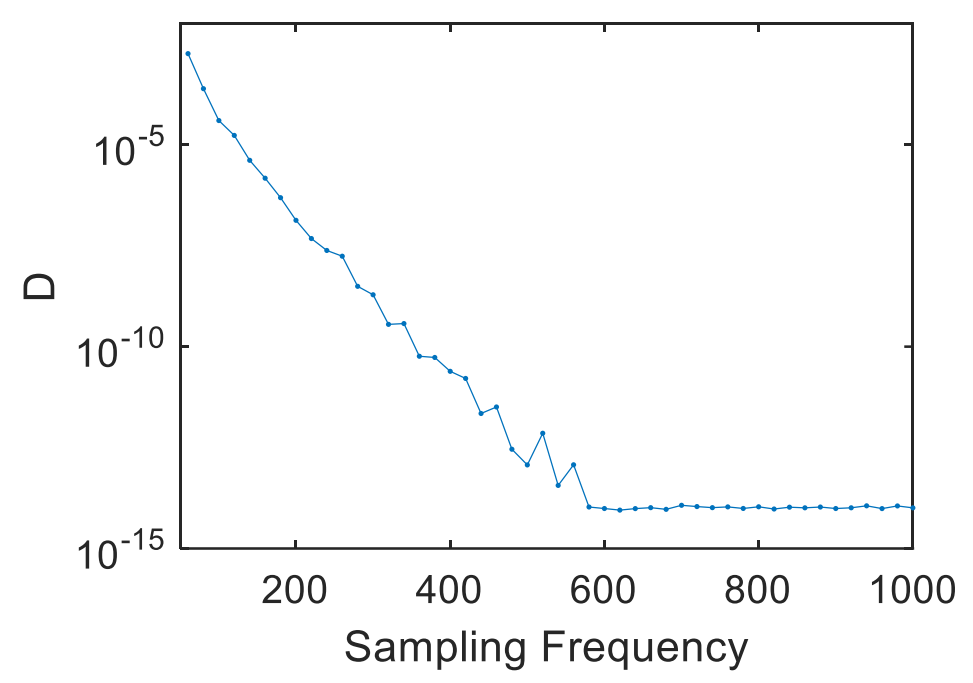

Fig. 10. Effect of sampling frequency on index $D$

\subsection{RHT for IF estimation}

In order to verify the performance of the RHT for IF estimation, the following signals are considered:

$$
\begin{aligned}
& \text { Signal 1: } x_{1}(t)=[1+0.1 \cos (2 \pi t)] \cdot \cos [4 \pi t+\sin (0.5 \pi t)] \\
& \text { Signal 2: } x_{2}(t)=[2+0.2 \cos (6 \pi t)] \cdot \cos [4 \pi t+\sin (0.5 \pi t)]
\end{aligned}
$$

The oscillation terms in signal 1 and signal 2 are the same, and the Ifs are identical, $f(t)=2+$ $0.25 \cos (0.5 \pi t)$. Yet the amplitudes of these two signals are different. Compared with the oscillating term, the amplitude function of signal 1 is a slow-varying signal, and the amplitude function of signal 2 contains higher frequency components. Therefore, the amplitude function and the oscillation term of signal 1 can be demodulated by the HT, but signal 2 can not be demodulated successfully.

In order to quantitatively demonstrate the estimation accuracy of different methods for estimating signal amplitude and IF, the error indexes are defined as

$$
I_{f}=\frac{\sqrt{\int_{0}^{T}\left[f_{\mathrm{id}}(t)-f(t)\right]^{2} \mathrm{~d} t}}{\sqrt{\int_{0}^{T}[f(t)]^{2} \mathrm{~d} t}}
$$




$$
I_{a}=\frac{\sqrt{\int_{0}^{T}\left[A_{\mathrm{id}}(t)-A(t)\right]^{2} \mathrm{~d} t}}{\sqrt{\int_{0}^{T}[A(t)]^{2} \mathrm{~d} t}}
$$

where $f_{\text {id }}$ and $A_{\text {id }}$ are estimated IF and amplitude function, respectively. The closer the estimated result is to the real result, the smaller the error indexes. The error indexes obtained by different calculation methods are shown in Tab.1. In Fig.11, the amplitude functions decomposed by several signal demodulation methods are shown and compared. The IF calculated by the above demodulation methods are compared in Fig.12.

For signal 1, the amplitude function error indicators calculated by the RHT and HT methods are 0.006 and 0.010 , respectively, and the IF error indicators are 0.013 and 0.017 , respectively. It is seen that the calculated value and the actual value are relatively close. The IF contains a remarkable error at two ends of the signal, which is caused by the end effect of the HT. In practical applications, the error caused by the end effect can be suppressed by signal mirroring or extension [27]. The amplitude function obtained by the TEAGER method is also close to the actual value but contains high-frequency fluctuations. The error indexes of the TEAGER method are greater than indexes obtained by the HT and RHT methods. In fact, the TEAGER method can only calculate accurate results when the signal amplitude and frequency are constant. The above discussion shows that for slow-varying signals satisfying the Bedrosian theorem, all three methods can get acceptable results. 
(a)

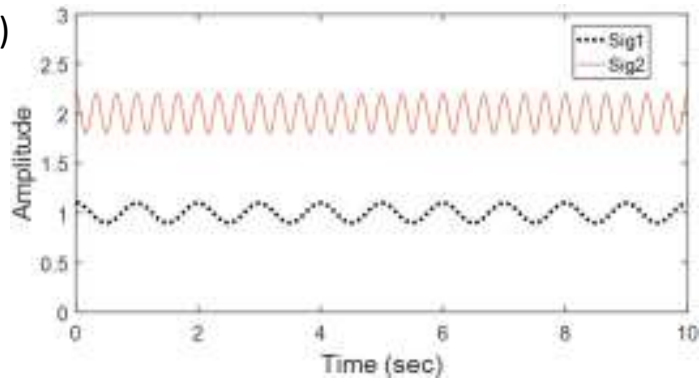

(c)

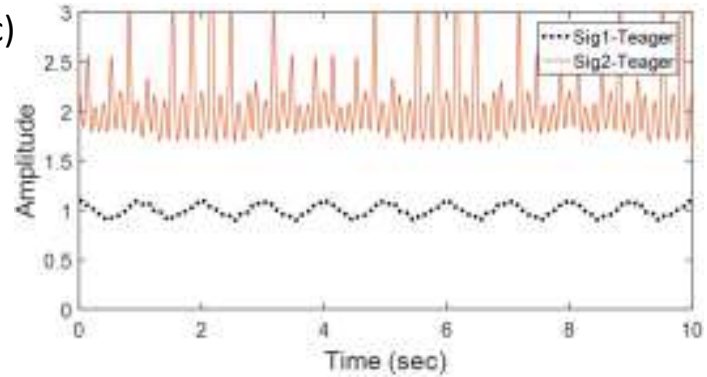

(b)

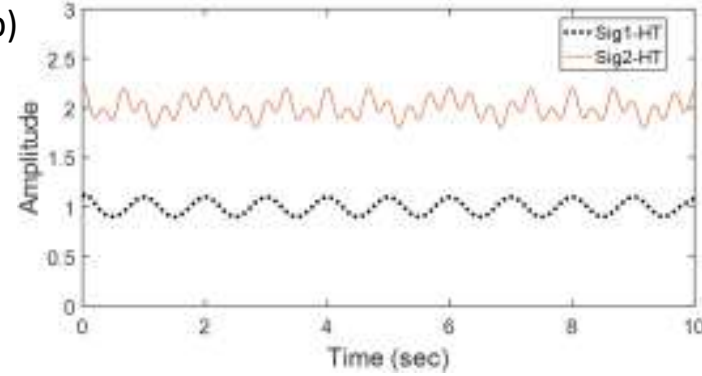

(d)

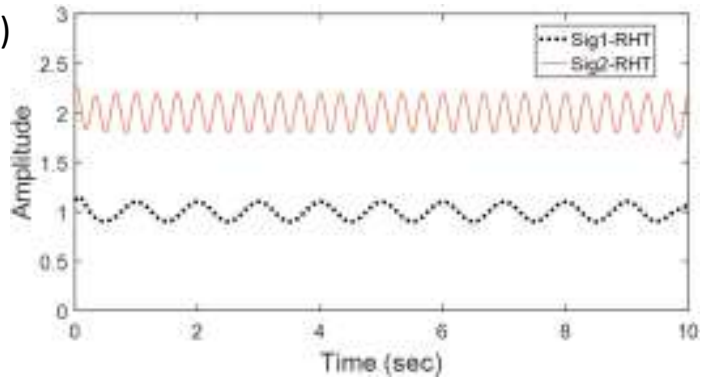

Fig.11 Comparison of the amplitude functions of signal 1 and signal 2 by different methods. (a): True value; (b): HT results; (c): TEAGER results; (d): RHT results.

(a)

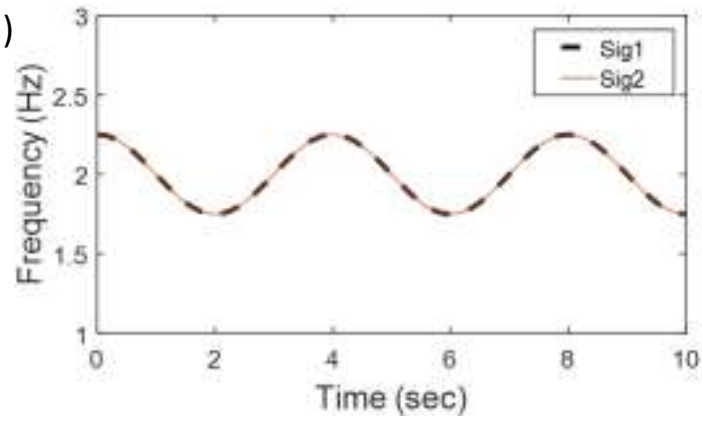

(c)

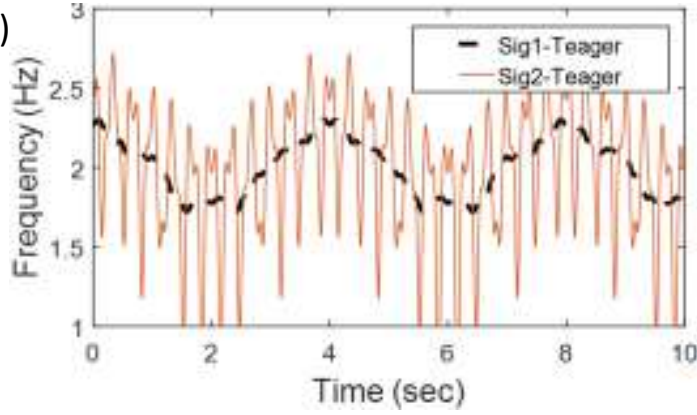

(b)

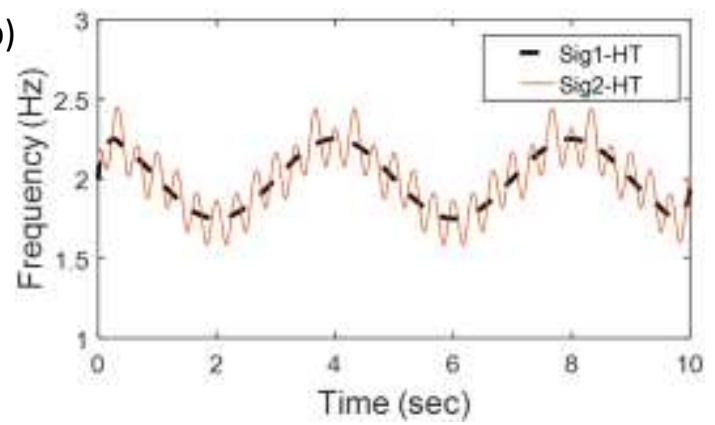

(d)

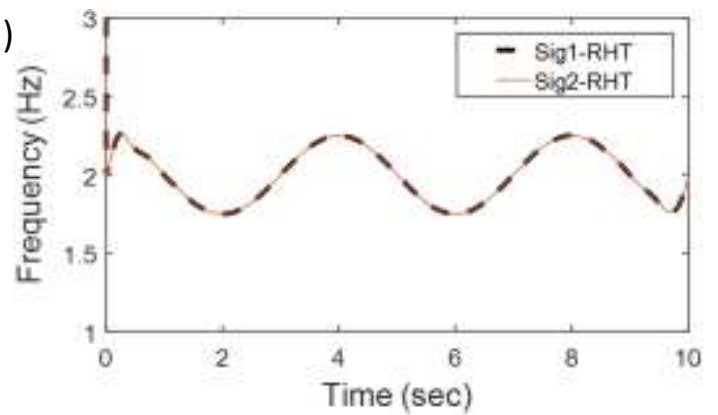

Fig.12 Comparison of the instantaneous frequencies of signal 1 and signal 2 by different methods. (a): True value; (b): HT results; (c): TEAGER results; (d): RHT results.

The amplitude function of signal 2 contains high-frequency components, thus the HT method cannot effectively demodulate such signals. The signal amplitude and IF obtained by the HT method are very different from the actual amplitude and IF, and the error indicators are increased to 0.051 and 0.058 
respectively. The TEAGER method also has large errors in amplitude and IF estimation. Therefore, neither HT nor TEAGER methods can effectively decompose the signal 2. It can be seen from the calculation results that only the RHT method can demodulate signal 2 accurately, and the error indexes are the same as the results of signal 1 . This reflects the stability of the RHT method and also shows that RHT is not restricted by the Bedrosian product conditions.

Tab.1 Error indexes of amplitude and frequency identification

\begin{tabular}{ccccc}
\hline \multirow{2}{*}{ Methods } & \multicolumn{2}{c}{$I_{a}$} & \multicolumn{2}{c}{$I_{f}$} \\
\cline { 2 - 5 } & Signal 1 & Signal 2 & Signal 1 & Signal 2 \\
\hline HT & 0.006 & 0.051 & 0.013 & 0.058 \\
TEAGER & 0.021 & 0.347 & 0.020 & 0.196 \\
RHT & 0.010 & 0.010 & 0.017 & 0.017 \\
\hline
\end{tabular}

\subsection{RHT for damped vibrating signal processing}

The damped free vibrating signal is the most common in vibration testing. A well-measured free vibration contains the damping ratio information and frequency information of the structure. For a long time, the use of HT to demodulate the damped vibration signals has been a common practice. The Fourier transform of the exponential decay function $f(t)=e^{-2 \pi f \xi t}$ is $\mathrm{F}(\omega)=\frac{1}{2 \pi f \xi+j \omega}$, where $\mathrm{t} \geq 0, \xi$ is the damping ratio and $f$ is the natural frequency of the system. Theoretically, when $\omega \rightarrow \infty, \mathrm{F}(\omega)$ is still greater than 0 . Therefore, a damped vibrating signal always violates the Bedrosian theorem. A simple damped vibrating signal is plotted in Fig. 13a, its damping ratio and frequency are 0.02 and $5 \mathrm{~Hz}$, respectively. In Fig.13b, both the Fourier amplitude spectrums of the amplitude function and the oscillation term are plotted. The amplitude function contains frequency components higher than the oscillation term. In this case, the signal violates the Bedrosian product theorem, which will cause a demodulation error in the HT. 

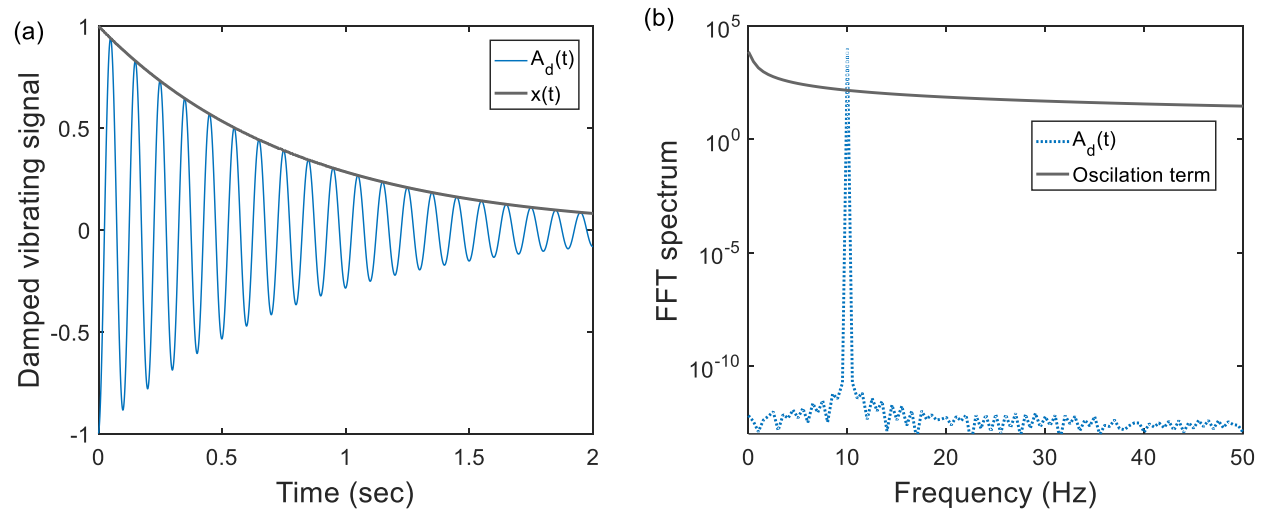

Fig.13 Damped Free vibration. (a): The damped vibration signal and its amplitude; (b): Fourier amplitude spectrums of the amplitude function and the oscillation term.

The extracted amplitudes from the HT and the RHT method are compared in Fig.14. The results show that at two ends of the signal, the amplitude function obtained by HT deviates from the real value; while the amplitude function given by RHT is closer to the real amplitude, but there are small glitches at some zero-crossing points. The glitches can be easily identified and eliminated in real applications. In Fig. 15, the Fourier transforms of the amplitude functions calculated by HT and RHT are compared. It can be seen that the result obtained by the RHT is closer to the true value. Therefore, this simple example shows that the RHT is more suitable for processing damped vibration signals.
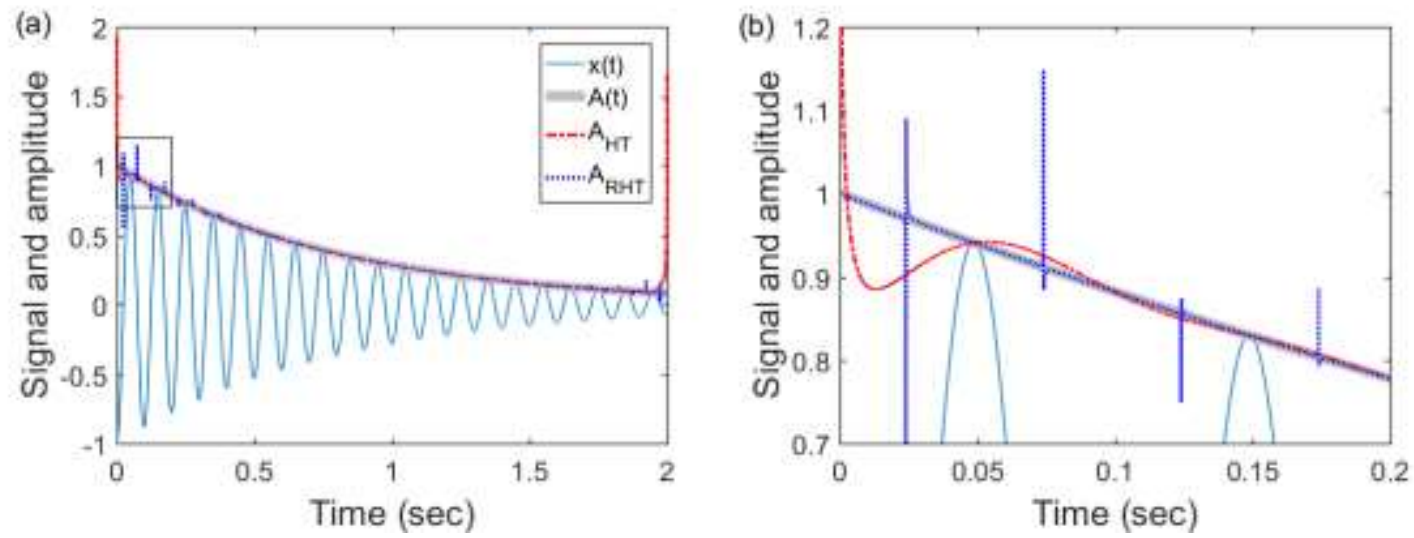

Fig.14 Demodulation results. (a): Amplitude result comparison; (b): Local zoon on demodulation results. 


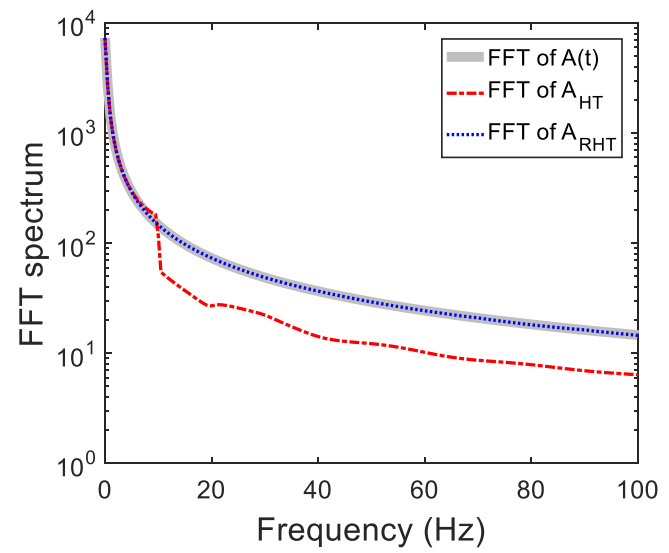

Fig.15 Fourier transform of the amplitude function obtained by HT and RHT

\section{Conclusion}

This article introduces the recursive Hilbert transform method for mono-component signal processing. For signals that do not satisfy the Bedrosian condition, the HT cannot accurately extract the amplitude and oscillation terms of the signal. The RHT method can overcome this problem. By analyzing the energy change of the quadrature error signal corresponding to the demodulated pure frequency modulation signal, we try to prove the convergence of the recursive process. The evolution of the discrete Fourier transform of the quadrature error signal is regarded as a vector autoregressive process. By proving that all singular values of the coefficient matrix of the autoregressive process are less than 1, the convergence of the RHT is guaranteed. A mathematical property about the eigenvalues of the tridiagonal Toeplitz matrices is used in the proof. However, since the proof of the convergence of RHT ignores the higher-order perturbation of the quadrature error signal, a rigorous proof of the convergence is still an open topic for future study.

On the other hand, the pure frequency modulation signal obtained by the RHT has the structure that its phase function $\phi_{K}$ makes $\exp \left(\mathrm{j} \phi_{K}\right)$ automatically becomes an analytical signal. The analytic signal with fixed zero-crossing points and constant amplitude seems to be unique, and the RHT can help us find out this analytic signal. Therefore, the RHT has important potential for analyzing analytic signals. In terms of application, since the RHT has the same zero-crossing position as the original signal, it can be known 
from the median theorem that it is suitable for demodulating and identifying signals with slow-varying instantaneous frequencies. The calculation examples in the paper also verified these application aspects.

\section{Abbreviations}

HT: Hilbert transform; IF: Instantaneous frequency; IA: Instantaneous amplitude; RHT: Recursive Hilbert transform; IMF: Intrinsic mode function; EMD: Empirical mode decomposition; HHT: Hilbert-Huang transform; HSD: Hilbert square demodulation; AM: Amplitude modulation; MC: Monte-Carlo;

\section{Declarations}

\section{Availability of data and materials}

All data generated or analysed during this study are included in this published article.

\section{Competing interests}

The authors declare that they have no known competing financial interests or personal relationships that could have appeared to influence the work reported in this paper.

\section{Funding}

The research is supported by the National Natural Science Foundation of China (grant no. 52178283), the Fundamental Research Funds for the Central Universities (grant no. JZ2021HGTB0103), and the Research Foundation of AnHui JianZhu University (grant no. 2019QDZ51).

\section{Authors' contributions}

ZXH conceptualized the problem, theoretically analyzed the algorithm, provided funds and compiled the manuscript. HYZ contributed to the theoretical analysis of the algorithm, and applied relevant software to visualize the problem. XH supervised the whole work, provided funds and participated in writing, commenting and editing. LHZ verified the proposed method and participated in writing, commenting and editing. All authors read and approved the final manuscript. 


\section{Acknowledgements}

We would like to express our gratitude to the editor and reviewers whose comments are of great help to improve the quality of this paper.

\section{Appendix A. Singular values of Matrix $A$}

Considering the characteristic of the matrix $\boldsymbol{A}$, let

$$
\boldsymbol{B}=\boldsymbol{A} \boldsymbol{A}^{\mathrm{T}}
$$

According to matrix theory, the singular values of the matrix are $\boldsymbol{A}$ the square roots of the eigenvalues of the matrix $\boldsymbol{B}$. The matrix $\boldsymbol{B}$ is a positive definite matrix, whose elements are given by

$$
\mathbf{B}_{i j}= \begin{cases}0.5 & \text { if } i=j \leq M-n_{2 \omega} \\ 0.25 & \text { if } i=j>M-n_{2 \omega} \\ -0.25 & \text { if }|i-j|=n_{2 \omega} \\ 0 & \text { else }\end{cases}
$$

The first $M-n_{2 \omega}$ diagonal elements equal to 0.5 , and the rest $n_{2 \omega}$ diagonal elements equal to 0.25 ; in addition, at the positions $(i, j)$, if $|i-j|=n_{2 \omega}$, the elements are -0.25 . A typical example of the matrix $\boldsymbol{B}$ is shown in Fig. A1, wherein $n_{2 \omega}=4, \mathrm{M}=10$. With a permutation matrix $\boldsymbol{P}$ the matrix $\boldsymbol{B}$ can be transformed into $\boldsymbol{B}_{d}$,

$$
\boldsymbol{B}_{d}=\boldsymbol{P} \boldsymbol{B} \boldsymbol{P}^{T}
$$

where the matrix $\boldsymbol{B}_{d}$ is a block diagonal matrix,

$$
\boldsymbol{B}_{d}=\left[\begin{array}{cccc}
\mathbf{J}_{1} & \mathbf{0} & \mathbf{0} & \mathbf{0} \\
\mathbf{0} & \mathbf{J}_{2} & \mathbf{0} & \mathbf{0} \\
\mathbf{0} & \mathbf{0} & \ddots & \mathbf{0} \\
\mathbf{0} & \mathbf{0} & \mathbf{0} & \mathbf{J}_{n_{2 \omega}}
\end{array}\right]
$$

Each block sub-matrix $\mathbf{J}_{i}$ in $\boldsymbol{B}_{d}$ is a tridiagonal Toeplitz matrix, which has the form 


$$
\mathbf{J}_{i}=\left[\begin{array}{ccccc}
a & b & \cdots & 0 & 0 \\
b & a & \cdots & 0 & 0 \\
\vdots & \vdots & \ddots & \vdots & \vdots \\
0 & 0 & \cdots & a & b \\
0 & 0 & \cdots & b & c
\end{array}\right]
$$

The elements in the matrix $\mathbf{J}_{i}$ are $a=0.5, b=-0.25, c=0.25$. Except for the last diagonal element $c=0.25$, the other diagonal elements are all 0.5 .

The permutation matrix can be obtained by rearranging the rows of the identity matrix, $\boldsymbol{P}=$ $\boldsymbol{I}\left(I_{p},:\right)$, where $\boldsymbol{I}$ is an $\mathrm{M} \times \mathrm{M}$ identity matrix, and $I_{p}$ is the index number of the row. In Fig. A1, the rearrangement of the matrix $\boldsymbol{B}$ is depicted and the index number is $I_{p}=[1,5,9,2,6,10,3,7,4,8]$. The matrix $\boldsymbol{B}$ is multiplied by $\boldsymbol{P}$ to the left and then multiplied by $\boldsymbol{P}^{T}$ to the right, the effect is the same as $\boldsymbol{B}_{d}=\boldsymbol{B}\left(I_{p}, I_{p}\right)$. Therefore, we can also directly use the method of changing the matrix index to calculate $\boldsymbol{B}_{d}$. In fact, in the RHT algorithm, there is no need for computing the matrix $\boldsymbol{B}_{d}$. Still, a formula for computing the index number is given below

$$
\operatorname{Ind}(k)= \begin{cases}\left\lceil\frac{k}{r+1}\right]+n_{\omega} \cdot \bmod (k-1, r+1) & k \leq m_{d}(r+1) \\ \left\lceil\frac{k-m_{d}(r+1)}{r}\right\rceil+m_{d}+n_{\omega} \cdot \bmod \left(k-m_{d}(r+1)-1, r\right) & k>m_{d}(r+1)\end{cases}
$$

wherein the intermediate variable $m_{d}$ and $r$ are given by

$$
\begin{gathered}
m_{d}=\bmod \left(N, n_{\omega}\right) \\
r=\left\lfloor\frac{N}{n_{\omega}}\right\rfloor
\end{gathered}
$$

The command $\bmod (a, b)$ returns the remainder after division of $a$ by $b,\lceil x\rceil$ and $\lfloor x\rfloor$ round $x$ towards positive and negative infinity, respectively. 
Because the permutation matrix is an orthogonal matrix, the eigenvalues of the matrix $\boldsymbol{B}$ are the same as the matrix $\boldsymbol{B}_{d}$. Furthermore, according to the mathematical result in [26], for an $\mathrm{M} \times \mathrm{M}$ tridiagonal Toeplitz matrix,

$$
\mathbf{J}_{i}=\left[\begin{array}{ccccc}
a & b & \cdots & 0 & 0 \\
b & a & \cdots & 0 & 0 \\
\vdots & \vdots & \ddots & \vdots & \vdots \\
0 & 0 & \cdots & a & b \\
0 & 0 & \cdots & b & a-\beta
\end{array}\right] \in R^{N \times N}
$$

Suppose $\beta=-b$, then the eigenvalues $\lambda_{k}$ are given by

$$
\lambda_{k}=a+2 b \cos \frac{2 k \pi}{2 M+1}, k=1,2, \cdots, M
$$

In our problem, nonzero elements of the matrix $\mathbf{J}_{i}$ are $a=0.5, b=-0.25, \beta=0.25$, then $a-\beta=$ 0.25. The eigenvalues are

$$
\lambda_{k}=0.5+0.5 \cos \frac{2 k \pi}{2 M+1}<1, \text { for } k=1,2, \cdots, M
$$

As shown in the above equation, each sub-block matrix $\mathbf{J}_{i}$ in the block diagonal matrix $\boldsymbol{B}_{d}$ has eigenvalues that less than 1, so all the eigenvalues of the matrix $\boldsymbol{B}_{d}$ are less than 1 . Thus, the eigenvalues of the matrix $\boldsymbol{B}$ are all less than 1. Since the singular values of matrix $\boldsymbol{A}$ are the square roots of the eigenvalues of the matrix $\boldsymbol{B}$, the singular values of matrix $\boldsymbol{A}$ are all less than 1. 


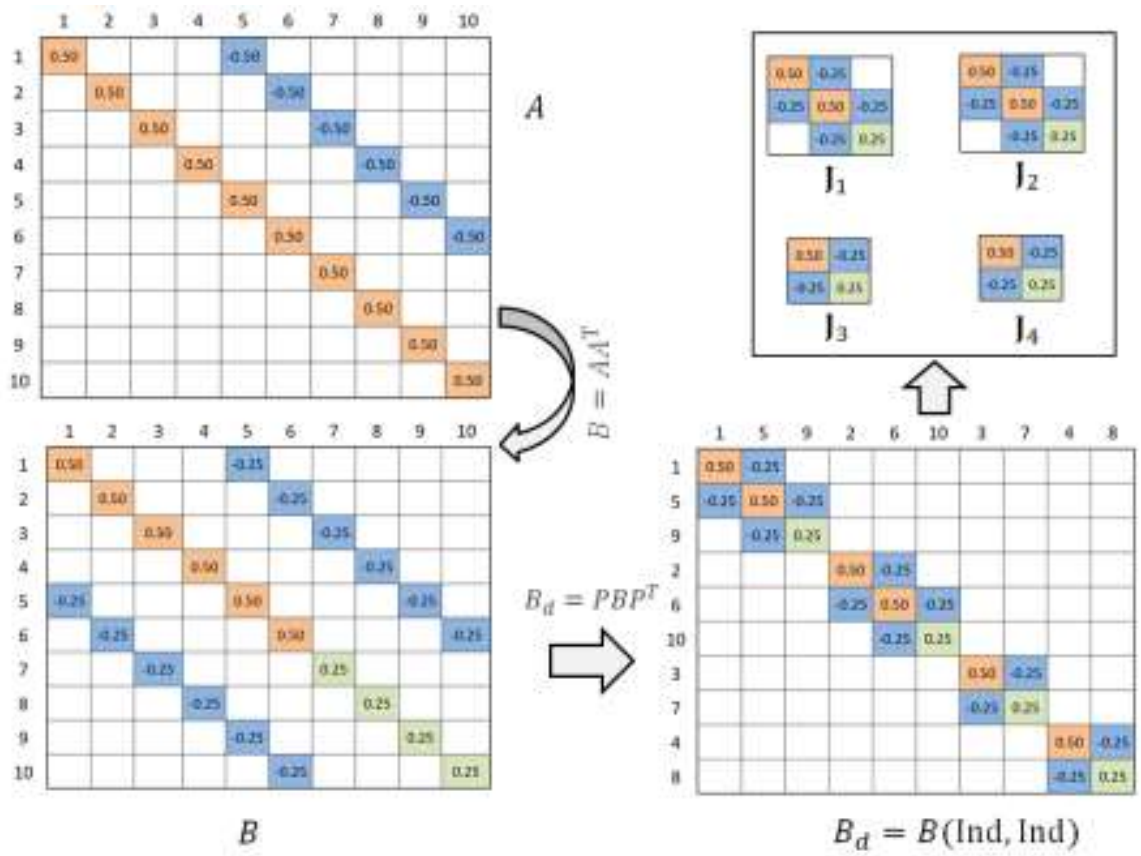

Fig. A1 Example matrices presenting the relationship of matrix $\boldsymbol{A}, \boldsymbol{B}, \boldsymbol{B}_{d}$, and $\boldsymbol{J} .\left(n_{2 \omega}=4, \mathrm{M}=10\right)$

\section{References}

[1] B. Picinbono, On instantaneous amplitude and phase of signals, IEEE Trans. Signal Process. 45 (1997) 552560. https://doi.org/10.1109/78.558469.

[2] P. Frank Pai, Instantaneous frequency of an arbitrary signal, Int. J. Mech. Sci. 52 (2010) 1682-1693. https://doi.org/10.1016/j.ijmecsci.2010.09.002.

[3] W. Lin, M. Xiaofeng, An adaptive Generalized S-transform for instantaneous frequency estimation, Signal Process. 91 (2011) 1876-1886. https://doi.org/10.1016/j.sigpro.2011.02.010.

[4] C. Wang, W.-X. Ren, Z.-C. Wang, H.-P. Zhu, Instantaneous frequency identification of time-varying structures by continuous wavelet transform, Eng. Struct. $52 \quad$ (2013) 17-25. https://doi.org/10.1016/j.engstruct.2013.02.006.

[5] I. Daubechies, J. Lu, H.-T. Wu, Synchrosqueezed wavelet transforms: An empirical mode decomposition-like tool, Appl. Comput. Harmon. Anal. 30 (2011) 243-261. https://doi.org/10.1016/j.acha.2010.08.002.

[6] N.E. Huang, Z. Shen, S.R. Long, M.C. Wu, H.H. Shih, Q. Zheng, N.-C. Yen, C.C. Tung, H.H. Liu, The empirical mode decomposition and the Hilbert spectrum for nonlinear and non-stationary time series analysis, Proc. R. Soc. Lond. Ser. Math. Phys. Eng. Sci. 454 (1998) 903-995. https://doi.org/10.1098/rspa.1998.0193. 
[7] Z. Wu, N.E. Huang, Ensemble empirical mode decomposition: a noise-assisted data analysis method, Adv. Adapt. Data Anal. 01 (2009) 1-41. https://doi.org/10.1142/S1793536909000047.

[8] D.P. Mandic, N. ur Rehman, Z. Wu, N.E. Huang, Empirical Mode Decomposition-Based Time-Frequency Analysis of Multivariate Signals: The Power of Adaptive Data Analysis, IEEE Signal Process. Mag. 30 (2013) 74-86. https://doi.org/10.1109/MSP.2013.2267931.

[9] M. Feldman, Time-varying vibration decomposition and analysis based on the Hilbert transform, J. Sound Vib. 295 (2006) 518-530. https://doi.org/10.1016/j.jsv.2005.12.058.

[10]S. Braun, M. Feldman, Decomposition of non-stationary signals into varying time scales: Some aspects of the EMD and HVD methods, Mech. Syst. Signal Process. 25 (2011) 2608-2630. https://doi.org/10.1016/j.ymssp.2011.04.005.

[11]F. Gianfelici, G. Biagetti, P. Crippa, C. Turchetti, Multicomponent AM-FM Representations: An Asymptotically Exact Approach, IEEE Trans. Audio Speech Lang. Process. 15 (2007) 823-837. https://doi.org/10.1109/TASL.2006.889744.

[12]Y. Qin, S. Qin, Y. Mao, Research on iterated Hilbert transform and its application in mechanical fault diagnosis, Mech. Syst. Signal Process. 22 (2008) 1967-1980. https://doi.org/10.1016/j.ymssp.2008.01.014.

[13] G. Chen, Z. Wang, A signal decomposition theorem with Hilbert transform and its application to narrowband time series with closely spaced frequency components, Mech. Syst. Signal Process. 28 (2012) 258-279. https://doi.org/10.1016/j.ymssp.2011.02.002.

[14]Z.-C. Wang, G.-D. Chen, Analytical mode decomposition of time series with decaying amplitudes and overlapping instantaneous frequencies, Smart Mater. Struct. $22 \quad$ (2013) 095003. https://doi.org/10.1088/0964-1726/22/9/095003.

[15] A. Potamianos, P. Maragos, A comparison of the energy operator and the Hilbert transform approach to signal and speech demodulation t, Signal Process. (1994) 26.

[16]M. Feldman, Hilbert transform in vibration analysis, Mech. Syst. Signal Process. 25 (2011) 735-802. https://doi.org/10.1016/j.ymssp.2010.07.018.

[17] E. Bedrosian, A product theorem for Hilbert transforms, Proc. IEEE. 51 (1963) 868-869. 
[18] Y. Xu, D. Yan, The Bedrosian identity for the Hilbert transform of product functions, Proc. Am. Math. Soc. 134 (2006) 2719-2728. https://doi.org/10.1090/S0002-9939-06-08315-8.

[19]E. Gengel, A. Pikovsky, Phase demodulation with iterative Hilbert transform embeddings, Signal Process. 165 (2019) 115-127. https://doi.org/10.1016/j.sigpro.2019.07.005.

[20] A.H. Nuttall, E. Bedrosian, On the quadrature approximation to the Hilbert transform of modulated signals, Proc. IEEE. 54 (1966) 1458-1459. https://doi.org/10.1109/PROC.1966.5138.

[21] N.E. Huang, Z. Wu, S.R. Long, K.C. Arnold, X. Chen, K. Blank, On instantaneous frequency, Adv. Adapt. Data Anal. 01 (2009) 177-229. https://doi.org/10.1142/S1793536909000096.

[22] J. Liu, J. Zheng, X. Wei, W. Ren, I. Laory, A combined method for instantaneous frequency identification in low frequency structures, Eng. Struct. 194 (2019) 370-383. https://doi.org/10.1016/j.engstruct.2019.05.057.

[23]B. Ge, Z.-C. Wang, Y.-J. Ding, Y. Mo, Hilbert square demodulation and error mitigation of the measured nonlinear structural dynamic response, Mech. Syst. Signal Process. 160 (2021) 107935. https://doi.org/10.1016/j.ymssp.2021.107935.

[24]Z. Hu, W. Ren, Vibration signal demodulaiton and instantaneous frequency estimation based on recursive Hilbert transform, J. Vib. Shock. 35 (2016) 39-43.

[25] Y. Shen, G.B. Giannakis, B. Baingana, Nonlinear Structural Vector Autoregressive Models With Application to Directed Brain Networks, IEEE Trans. Signal Process. $67 \quad$ (2019) 5325-5339. https://doi.org/10.1109/TSP.2019.2940122.

[26] W.-C. Yueh, Eigen values of several tridiagonal matrices, Appl. Math. E-Notes. 5 (2005) 66-74.

[27] D.-C. Lin, Z.-L. Guo, F.-P. An, F.-L. Zeng, Elimination of end effects in empirical mode decomposition by mirror image coupled with support vector regression, Mech. Syst. Signal Process. 31 (2012) 13-28. https://doi.org/DOI: 10.1016/J.YMSSP.2012.02.012. 